\title{
Campaign rhetoric and the hide-and-seek game
}

\author{
Sourav Bhattacharya ${ }^{1}$
}

Received: 15 November 2012 / Accepted: 11 August 2016 / Published online: 15 September 2016 C The Author(s) 2016. This article is published with open access at Springerlink.com

\begin{abstract}
We present a model of political campaigning where a candidate chooses between promoting oneself (positive campaign) or attacking the rival (negative campaign). Candidates vary only by quality. Campaign choices determine the subject of public deliberation: If a candidate runs a positive campaign and his rival a negative campaign, the voters learn the quality of the "focal" candidate. Thus, negative campaigns may be used either to expose the rival candidate (informative role) or to turn attention away from oneself (non-informative role). The effect of negative campaigns depends on whether it is faced with another negative campaign (cross talk) or a positive campaign (fruitful debate). We suggest that in order to ascertain the effect of negative advertising, studies should take into account the campaigns employed by both candidates. Voter beliefs about candidate quality plays a major role in campaign selection: while the incidence of negative campaigning goes down as the prior probability of a candidate being good increases, the probability of selection of the correct candidate is non-monotonic in the said prior.
\end{abstract}

\footnotetext{
I thank David Austen-Smith, Allen Brierly, Steve Callander, Joyee Deb, Alexandre Debs, Tim Feddersen, Yuk-Fai Fong, Sean Gailmard, Bard Harstad, Jaehoon Kim, George Krause, Tapas Kundu, Marciano Siniscalchi, seminar participants at MPSA conference (2006), Michigan State University and CIDE, Mexico for comments and suggestions. I also acknowledge the hospitality and financial support from Wallis Institute of Political Economy, University of Rochester that I enjoyed while preparing part of the manuscript. Any error that remains in the paper is my responsibility.
}

Electronic supplementary material The online version of this article (doi:10.1007/s00355-016-0988-6) contains supplementary material, which is available to authorized users.

$凶$ Sourav Bhattacharya

sourav.bhattacharya@rhul.ac.uk

1 Department of Economics, Royal Holloway University of London, Egham, UK 


\section{Introduction}

Electoral campaigns are possibly the most important element of voters' information about the candidates. However, campaigns do not contain disinterested, impartial information. Candidates choose their rhetoric strategically in order to influence voter perception. One aspect of such strategic choice that has received considerable attention both in the media and in the academy is the choice between positive and negative advertising: Whether to highlight one's own quality or to focus on why the rival is unsuitable for office. While every electoral campaign involves a large number of positive, negative and (especially) comparative messages, we shall characterize the overall theme of a campaign as broadly positive or negative, and it is the choice of this theme that we shall examine in this paper. ${ }^{1}$

In this paper, we think of campaigns as public debates in which the voting public forms its judgement about the candidates by comparing and contrasting their campaign statements. Thus, the nature and extent of information revealed about candidates depends jointly on the campaign themes chosen by both candidates. In particular, our main premise is that if one candidate decides to highlight why he is suitable (positive campaign) and the other argues why his rival is unsuitable (negative campaign), then the former candidate is the focal one in the electoral race: The public learns more about quality of the former than about the latter one. This creates the main tension that we study in the paper: Good candidates try to reveal information through focality and the bad ones trying to avoid focality. The analysis borrows its basic idea from the literature on issue choice in campaign rhetoric (Simon 2002; Kaplan et al. 2006; Egorov 2012): More information is revealed about an issue if the two candidates discuss both sides of the issue rather than if only one candidate presents his side of the issue. In this paper, we study the candidate's decision to focus on one of two decidedly aggregate issues: Matters pertaining to one's own suitability for office vs. those pertaining to the rival's quality. Such a model allows us to analyze conditions under which negative advertising is used to inform the electorate or to muddle the debate.

Opinions are deeply divided regarding whether the practice of negative campaigning improves or degrades the electoral process. While some view negative campaigns as essentially vicious mud-slinging with the effect of reducing turnout (Ansolabehere and Iyengar 1995; Ansolabehere et al. 1994, 1999), destroying voters' trust in government and undermining the efficacy of the political system (Brader 2005), others (Geer 2006) have pointed out its role in providing genuine information and disciplining the candidates, and even in stimulating turnout (Freedman and Goldstein 1999, 2002; Finkel and Geer 1998; Kahn and Kenney 1999; Wattenberg and Brians 1999). Over the last two decades, there has been a profusion of empirical and experimental research examining these effects. However, in a meta-study covering 110 articles, dissertations and books published on the topic between 1987 and 2007, Lau et al. (2007) finds that

\footnotetext{
1 While some of the literature has looked at the role of individual messages, a large chunk of the empirical literature has "coded" campaigns as either primarily positive or primarily negative or the extent to which a whole campaign is negative. Ansolabehere et al. (1994, 1999), in their seminal study of turnout in 34 senate elections in 1992, use this binary characterization. Others (e.g., Brooks 2006; Wattenberg and Brians 1999) have used the same data.
} 
there is no consensus in the literature on the role of negative advertising (see also Lau et al. 1999). All empirical or experimental work aimed at uncovering the role of negativity in advertising has so far looked at the effect of individual messages/campaigns in isolation - our model postulates that we may get a clearer and more nuanced picture if future work takes into account both the campaign themes (or messages) in contention.

There is a wide variation in the definition of negative advertising employed in the literature. At one extreme, Ansolabehere and Iyengar (1995) equates negative advertising with mudslinging and ad hominem attacks; and at the other extreme, Polborn and Yi (2006), henceforth referred to as PY, has defined negative advertising exclusively as provision of valuable electoral information about the rival candidate. We use a broad definition of negative and positive campaigns that is adopted from Finkel and Geer (1998): "[P] ositive appeals are ones that candidates offer to promote themselves on some issue or trait"; and "Negative appeals are attacks [or criticism] leveled at the opposition." While this definition literally applies to specific messages, we adjust this definition for aggregate campaign themes. In our model, a positive campaign may contain messages about one's own strength of character, values, records and arguments about why one's position on different issues is close to that of the median voter. On the other hand, a negative campaign may contain a series of messages regarding the opponent's features (character flaws, vices, inconsistencies, past failures) and arguments why his political positions are not close to that of the median voter. In sum, the crucial difference between a positive and negative campaign is whether the informational spotlight is focused on oneself or on the rival. According to this definition, there is nothing inherently negative about talking about the rival (or positive about focussing on oneself). However, it is never in the interest of a candidate to highlight his own failings or his rival's virtues: Typically, self-focussed campaigns contain positive information and rival-focussed campaigns contain negative information.

Given this categorization of campaign themes, whether negative advertising is useful or harmful to the voter arises endogenously from candidate choices. When one candidate goes positive and the other negative, the former candidate's quality is the focal issue: The candidates engage in an active debate about this issue. The voters learn about the focal candidate by comparing claims and counterclaims. Most of the preelectoral discussions in the public sphere are centered on the focal candidate. Three recent examples of focal candidates in high profile elections are Barack Obama in the American Presidential Election of 2008, Narendra Modi in the Indian parliamentary (Lok Sabha) election of 2014, and Donald Trump in the American Presidential race of 2016. Compared to the focal candidate, a lot less is learnt about the rival in such elections. Not every election has a focal candidate, however. If both candidates run positive campaigns or both employ negative campaigns, they are essentially "talking past each other". Since there are only one-sided claims which are less credible, the voters learn a lot less about either candidate in this case.

As the campaign watchdog, the media has an important role to play in our story. Following PY, we think of the candidates as setting the agenda for the media through their choice of campaign themes. The media checks the veracity of statements made by the candidates, compares claims and counterclaims and produces a judgement on the issue being debated. Given the constraints on the time, space and resources of the 
media, fact-checking more efficient when there is concentrated engagement about one candidate rather than a diffused discussion about both candidates.

Scholars studying American political campaigns have long stressed the need for issue engagement (or "dialogue") for enabling the voting public to make an informed decision. Berelson Bernard et al. (1954), Kelley (1960), and more recently Petrocik (1996) and Sellers (1998) document and bemoan the tendency of candidates to talk past each other. Simon (2002) defines "dialogue occurs only when two candidates address the same subject ... the opposite of dialogue is ignoring, responding by discussing a different subject". Emphasizing that "the level of dialogue approximates the quality of the campaign", he goes on to observe that the "decision to dialogue can be construed as a strategic choice". This strategic choice is formally modelled by Simon (2002), Kaplan et al. (2006) and Egorov (2012) where they assume that voters learn more about issues over which candidates choose to engage in a dialogue. Simon (2002) and Kaplan et al. (2006) also estimate the extent of dialogue over different issues in several empirical and laboratory settings. In the current paper, we extend the logic of issue choice to the specific case of positive vs. negative campaign themes.

Geer (2006), in his detailed historical study of American Presidential campaigns, informally makes an argument similar in spirit to what has been modeled in this paper. While positive campaigns allow candidates to "talk past each other" and voters would "assume innocence unless proved guilty", negative campaigns carry the risk of being caught "making unsubstantiated charges" (Geer 2006; p. 51). We allow, in addition, the possibility that even negative campaigns, when carried on by both sides, may not reveal much information either.

This paper takes seriously the fact that candidates often spend large amount of resources on espionage. Even if a candidate himself is well-known to the voting public, there may be aspects about his suitability to office that are not always well known. Thus, each campaign spends time and money in finding negative information about the rival. Such efforts capture a range of things from researching the rival candidate's voting records and personal history to privately polling sections of the electorate in order to find out about how the public views the rival's positions on salient issues. We embed in our model two features of such espionage: First, it is costly, and second, it is almost always clandestine. ${ }^{2}$

\subsection{Model: brief discussion}

In our formal model, each of the two candidates has a privately known quality - either good or bad. At the beginning of the campaign, each candidate has the option to learn about the quality of the rival by investing a cost. Armed with information about their own quality and possibly the rival's quality, candidates commit to a campaign theme: Positive or negative. Such precommitment is a simplifying assumption made in order to ignore the dynamic aspect of campaigns where a candidate makes counter-claims in response to the rival's claims, and is entirely standard in the literature on issue choice. In the model, we blackbox the actual process of voter opinion formation in what we

2 For instance, here is an article about clandestine political espionage network for the Republican cause in the United States: http://www.politico.com/story/2015/11/the-koch-brothers-intelligence-agency-215943. 
call the information revelation protocol. If one candidate chooses a positive campaign and the other a negative one, we say that there is a fruitful debate. Otherwise, there is cross talk. In case of a fruitful debate, the candidate whose quality is discussed is said to be focal while the other candidate is non-focal. In case of cross-talk, neither candidate is focal. In our benchmark case, we assume a deterministic information revelation protocol: a candidate's true quality is revealed if and only if he is focal, and the campaign reveals no information about non-focal candidates. In a latter extension, we assume a probabilistic information revelation protocol: the quality of focal the candidate is revealed with a higher probability than that of the non-focal candidate. Voters vote based on information revealed through the protocol under consideration. All voters have the same preference and prefer to vote for the good rather than the bad candidate.

Given the information revelation protocol, if a good candidate faces a bad candidate, the former would prefer that either candidate be focal while the latter would prefer that neither is focal. This means that the two candidates have opposite preferences over message profiles: The good candidate prefers to go positive (negative) when the bad candidate goes negative (positive), and the bad candidate prefers to go positive (negative) when the good candidate goes positive (negative), leading to a matching pennies game between the good and bad types. Since the bad candidate wants to hide (conceal information) and the good candidate wants to chase (reveal information), we title the competitive process of information provision as a hide-and-seek game. The unique equilibrium outcome of this game describes candidate behavior and determines the extent of information revealed in electorate.

The introduction of uncertainty between candidates and the option of costly search is a crucial innovation in this paper. Between-candidate uncertainty captures a fundamental asymmetry between positive and negative campaigns that cannot be obtained in models which start out assuming that candidates know about each others' type (e.g., PY). While information used in a positive campaign is free for a candidate, truth in a negative campaign is obtainable only through costly research. As a consequence, positive campaigns contain more information than negative campaigns.

\subsection{Main results}

Because of incomplete information between candidates, the voters' prior belief about a candidate being good plays a major role in determining the nature of debate. Using this prior as a proxy for average (expected) candidate quality, we focus on understanding the strategic behavior of candidates as a function of expected candidate quality. The model confirms the conventional wisdom that the incidence of negative advertising increases as the average quality worsens. However, the hide-and-seek nature of the race provides an advantage to the "rarer" type. In particular, a good candidate can most effectively reveal his type through positive advertising when the average candidate quality is low and there is a lot of negative campaigning. At the other extreme, when the average candidate quality is very high, all candidates engage in more positive campaigning: But this allows the bad candidates to effectively "hide" by avoiding debate with the good types. Thus, while an electorate with low expected candidate quality is competitive but efficient for the purpose of candidate selection, one with a 
high average quality is inefficient. Consequently, voter welfare in terms of the ex-post expected quality of the winning candidate may actually go down with an increase in the ex-ante expected candidate quality.

The model also allows us to talk about a novel role of campaign resources. Snyder (1989), for example, examines the role of campaign resources in finding information about the opponent. The search cost can be seen as an index of the extent of asymmetry in information between the candidates. While a lowering of this cost improves the information content of negative advertising, we show that the bad type has a stronger incentive to engage in search than the good type. Therefore, the expected welfare of the voter is not monotonic in the cost of search. In particular, if a social planner could "select" the cost of search in order to maximize the probability of correct selection, then she would set the cost to zero when the good type is more common and set the cost very high when the bad type is more common. Similarly, the voter's welfare is not monotonic in the payoff from winning office.

\subsection{Related formal work}

As mentioned before, the current paper brings together two strands of theoretical work on political campaigns: One on issue engagement and the other on positive vs. negative advertising. Simon (2002) presents a model where candidates allocate an advertising budget over many issues, and shows that each candidate has an incentive to emphasize a different set of issues rather than engaging in a dialogue with the rival on any given issue. According to Simon, a dialogue can be expected on an issue only if (i) it has overriding salience, (ii) mass media forces dialogue, or (iii) candidates behave "irrationally" for whatever reason. Kaplan et al. (2006) introduces uncertainty about voter preferences over candidates' positions and obtains dialogue in Simon's model in a mixed strategy equilibrium. In our model, the between-candidate uncertainty performs a similar role as candidate uncertainty about voter preferences in Kaplan et al. (2006) and leads to fruitful debate in equilibrium.

Earlier formal work on choice between positive and negative advertising (Skaperdas and Grofman 1995; Harrington and Hess 1996) takes as primitive an "influence function" that assumes that negative advertising reduces support both for its sponsor and target. ${ }^{3}$ Skaperdas and Grofman (1995) finds that the trailing candidate engages in more negative advertising than the frontrunner, and Harrington and Hess (1996) finds that the candidate with less attractive personal attribute runs a more negative campaign. In the equilibrium of our model too, the "bad" candidate is weakly more likely to engage in a negative campaign than the "good" candidate (and strictly so unless the prior expected candidate quality is very high).

PY considers a signaling model where candidates rationally choose the campaign theme and voters infer candidate quality by observing the chosen theme. ${ }^{4}$ The authors find that a candidate uses a negative campaign when he either does not have too much

\footnotetext{
3 Desposato (2008) studies the incidence of negative advertising in Latin American countires in a comparative perspective by using similar influence functions.

4 Mattes (2007) is a very similar model with choice between informative advertising on two dimensions: negative vs. positive and issue vs. character.
} 
to say about himself or he knows that his opponent is very bad. While these authors assume that negative advertising contains only hard information and is therefore always informative, our framework accounts for both "good" and "bad" motivations for attack by considering softer information. Consequently, these two models generate opposite empirical predictions about the effectiveness of negative advertising.

PY finds that in an environment where most candidates are expected to run negative campaigns, when a candidate runs a negative campaign against one running a positive campaign, the former is more likely to win. We obtain the exactly opposite conclusion: in such an environment, the positive campaigner has an advantage. In our model, when the expected candidate quality is very low, the bad types use negative campaigns to turn the focus on the rival-but this allows the rare good type the opportunity to create focality by running a positive campaign.

We also show that the full knowledge of rival type assumed in PY does not hold, even for very small search costs.

The remainder of the paper is organized as follows. Section 2 sets up the benchmark model in which the candidates are either "good" or "bad" and Sect. 3 characterizes the equilibrium. Section 4 discusses how candidate behavior in the benchmark case changes as the prior belief and the search cost change, and analyses welfare implications. Section 5 presents a few extensions and Sect. 6 concludes the main body of the paper. The proof of the main proposition (Proposition 1) is presented in the appendix. The supplementary online material discusses an extended version of the model with voter inference and a more general type space.

\section{Basic model: binary types}

There are two players: Candidates $i=1$ and $i=2$, each with a private quality $\theta_{i},(i=1,2)$. Quality $\theta_{i}$ can be either $\operatorname{Good}(G)$ and $\operatorname{Bad}(B) .{ }^{5}$ For both candidates, quality follows a commonly known Bernoulli distribution with the prior probability of a good type $\alpha \in(0,1)$. The candidates run campaigns (positive or negative) which reveal information to a voter about quality, based on which she votes for one of the two. While the voter is not modeled explicitly as a player, her actions based on the revealed information are taken into account in the payoffs arising from candidate actions. In the supplementary online appendix we show exactly the same conclusions hold when we formally model the voter as a player.

\subsection{Actions}

Player $i$ has two different action choices: The campaign action $M_{i} \in\{P, N\}$ and the search action $X_{i} \in\{S, N S\}$. $P$ denotes a positive campaign and $N$ denotes a negative

\footnotetext{
5 The "quality" of a candidate can be thought of in two ways. In a common values framework where all voters have the same preference, quality captures all characteristics that voters care about. In a private values framework where the two candidates can be assigned two locations on the left-right ideological continuum, quality can simply be thought of as the distance of a candidate's location from the median voter's ideal point.
} 
one. If player $i$ undertakes action $S$ (search), he gets to know the type of his rival $-i$ with certainty. If action $N S$ (no search) is taken, the rival's type is not known. One can think of the search action as being taken before the campaign choice, so that the message can be conditioned on the information obtained through search. We assume that the search action itself is private, i.e., a candidate cannot detect whether the rival has searched or not. Thus, the search stage and the debate stage can be considered simultaneous from the strategic point of view. Campaign choices by the candidates are also, in the same sense, strategically simultaneous: We assume that a candidate cannot condition his choice of message on the message chosen by the other candidate. ${ }^{6}$ The campaign profile is denoted by $\mathbf{M}=\left\{M_{1}, M_{2}\right\}$, the search profile by $\mathbf{X}=\left\{X_{1}, X_{2}\right\}$ and the type profile by $\boldsymbol{\theta}=\left\{\theta_{1}, \theta_{2}\right\}$. Campaigns are thought of as public debates. The payoff to player $i$ from an action and type profile $\{\mathbf{M}, \mathbf{X}, \boldsymbol{\theta}\}$ is assumed to be the payoff $u_{i}(\mathbf{M}, \boldsymbol{\theta})$ from debate less the cost of search, which is $c>0$ if $X_{i}=S$ and zero otherwise. The payoff from debate $u_{i}(\mathbf{M}, \boldsymbol{\theta})$ depends on the information revelation protocol. The winner of the debate gets a payoff $w>0$, while the one who loses the debate gets a payoff of 0 . We normalize $w=2$.

The search cost $c$ is an index of how easy it is for a candidate to find out detailed information about the other candidate: In this sense it measures the extent of asymmetry of information between candidates. Since the payoff from winning the debate has been normalized, in effect, $c$ captures the ratio of the actual cost of search to the payoff from winning office. Thus, an increase in the importance of the contested office (with the difficulty of finding information about the rival remaining the same) would imply a drop in search cost. In the paper, we restrict $c$ to lie in the range $\left(0, \frac{1}{2}\right)$. In this range, we show that search occurs in equilibrium for moderate values of the prior $\alpha \in(c, 1-c)$. While the case with $c>\frac{1}{2}$ can easily be covered by the model, we do not formally study this case since there is no information acquisition for any prior belief over candidate quality in equilibrium (please see footnote 9).

\subsection{Information revelation protocol}

The campaign can be thought of as a public debate that reveals information to the voter. The actual process of debate is not modeled: We assume a protocol which determines how information about candidate types is revealed to the voter depending on the campaign profile. If one candidate goes positive and the other goes negative (i.e., $M_{1} \neq M_{2}$ ), then the former candidate is focal in the sense that his quality is subject to a fruitful debate. If on the other hand, there is cross talk, i.e., if $M_{1}=M_{2}$, there is no focal candidate. We assume that a focal candidate's quality is revealed to the voter and a non-focal candidate's quality is not.

A focal candidate wins the election if he is revealed to be good and loses the election if he is revealed to be bad. If there is cross talk, the winner is chosen randomly with equal probability. Although simplistic, such a passive voter response to debate works

\footnotetext{
6 Since the campaign theme is part of the broader campaign strategy which is determined in advance (rather than tactics which can change as the campaign progresses), the simultaneity of message choice is not too bad an assumption to start with.
} 
for a two-type case because if one candidate is revealed to be good (bad), the other candidate cannot be better (worse). In the online appendix, we show that if we include the voter as a rational player in the game, such "passive" voting strategies considered here arise as equilibrium behavior. Assume that the winner of the debate (i.e., whoever the voter votes for) gets a payoff normalized to 2 . In this constant sum game, the utility from debate $u_{1}(\mathbf{M}, \boldsymbol{\theta})$ for player 1 is given by the following table, and that for player 2 is $u_{2}(\mathbf{M}, \boldsymbol{\theta})=2-u_{1}(\mathbf{M}, \boldsymbol{\theta})$.

$$
\begin{aligned}
u_{1}(P, P, \boldsymbol{\theta}) & =u_{1}(N, N, \boldsymbol{\theta})=1, \\
u_{1}\left(P, N, G, \theta_{2}\right) & =u_{1}\left(N, P, \theta_{1}, B\right)=2, \\
u_{1}\left(P, N, B, \theta_{2}\right) & =u_{1}\left(N, P, \theta_{1}, G\right)=0,
\end{aligned}
$$

The rather extreme assumption that the media reveals the true type of the focal candidate and reveals nothing otherwise is not necessary for the qualitative results of the model. It has been made for technical convenience so as to be able to drive the basic point home without using unnecessary parameters. In Sect. 5 we discuss the results in a generalized model where this extreme assumption is relaxed while retaining the feature that a claim reveals information with a higher probability if it is faced with a counter-claim. In that section, we use a probabilistic (rather than deterministic) information revelation protocol which assumes that (1) in a fruitful debate, the quality of the focal candidate is revealed with a higher probability than the non-focal one, and (2) the probability that the voter learns neither candidate's type is higher under cross talk than under a fruitful debate.

\subsection{Incentives}

To understand the incentives that such a payoff structure creates, it is useful to describe them in a normal form. There are three possible situations, depending on whether a good type faces a good type, a bad type faces another bad type, or whether the two

\begin{tabular}{|c|c|c|c|}
\hline \multicolumn{4}{|c|}{ B-type } \\
\hline \multirow{3}{*}{ G-type } & & & $\mathrm{N}$ \\
\hline & $\mathrm{P}$ & 1 & 2 \\
\hline & $\mathrm{N}$ & 2 & 1 \\
\hline
\end{tabular}
candidates are of different types. Each case is described in a separate $2 \times 2$ matrix, with the row player's payoff shown in the matrix.

$$
\begin{aligned}
& \text { G-type } \begin{array}{|r|r|r|}
\multicolumn{1}{c}{\text { G-type }} \\
\hline \mathrm{P} & \multicolumn{1}{c}{\mathrm{P}} & \mathrm{N} \\
\cline { 2 - 3 } & 1 & 2 \\
\cline { 2 - 4 } & 0 & 1 \\
\hline
\end{array} \\
& \text { B-type } \begin{array}{|c|c|c|}
\multicolumn{1}{c}{\text { B-type }} \\
\hline \mathrm{P} & \mathrm{P} & \mathrm{N} \\
\mathrm{N} & 1 & 0 \\
\cline { 2 - 4 } & 2 & 1 \\
\hline
\end{array}
\end{aligned}
$$


Suppose for now the candidates knew each other's types. Then they would know which one of the above three situations they were in. If a good candidate were facing another good candidate, then it is strictly dominant for both to run a positive campaign: Both players would want to discuss their own qualities. If a bad candidate were facing another bad one, then it is strictly dominant to use a negative campaign: Neither player wants the focus on himself. When a good candidate faces a bad candidate, there is a matching pennies game: The good candidate wants to reveal information about either candidate: Thus he wants either to be focal himself $(P, N)$ or the rival to be focal $(N, P)$. The bad candidate, on the other hand, wants to conceal information by inducing cross talk $(P, P)$ or $(N, N)$. What makes this game interesting is that there is incomplete information about the rival's type, and each candidate has an incentive to invest in research about the rival's quality before deciding the campaign theme.

At this point, two comments are in order about the assumptions of the model.

In a formal sense, the campaign choice $M_{i}$ by candidate $i \in\{1,2\}$ is a message about his private information $\theta_{i}$. There are two traditions in economics about the relationship between messages and private information, and both have been applied to the analysis of political advertising. At one extreme, messages are thought to be cheap talk, i.e., completely unverifiable (Prat 2004). At the other extreme, messages are considered to be completely verifiable "signals" about private information, and therefore by definition truthful (PY). We recognize that while the content of candidate statements often carry valuable information about candidate types, such statements also include half-truths, unverifiable claims and outright lies. Even a fact-checking media may not always be able to ascertain the truth in all cases. We therefore assume that messages are only conditionally verifiable, i.e., $\theta_{i}$ is revealed only when $M_{i}=$ $P$ and $M_{-i}=N$. One interpretation could be that there is always two kinds of information about a candidate: Positive information and negative information. Very often, both kinds of information have to be weighed against each other to have full picture of the quality of a candidate. Such a complete assessment cannot be obtained if both sides of the same issue are not debated by the candidates.

We assume that search by one candidate about the other's quality is private. There are two restrictions embedded in this assumption. First, while deciding on the campaign theme, a candidate does not know whether his rival has found the relevant information to ascertain his quality. Second, when there is a fruitful debate, the voting public cannot distinguish whether the negative facts about the focal candidate were unearthed by the media or the rival candidate or were simply revealed through the process of debate. See footnote 10 for how the results would change when search is still a costly option, but a candidate is aware of whether the rival searched or not.

\section{Equilibrium and its properties}

The equilibrium concept considered here is Bayesian Nash equilibrium. Formally, the strategy set for type $\theta \in\{G, B\}$ of a candidate $i \in\{1,2\}$ consists of the following three elements:

1. $p_{i}(\theta)$ : probability of search.

2. $q_{i}(\theta)$ : probability of using a positive campaign $(P)$ conditional on not searching. 
3. $r_{i}\left(\theta, \theta^{\prime}\right)$ : probability of type $\theta$ of candidate $i$ using a positive campaign $(P)$ conditional on searching, and discovering candidate $j$ to be of type $\theta^{\prime}$.

From the previous discussion, we must have $r_{i}(G, G)=1$ and $r_{i}(B, B)=0$ (strictly dominant strategies). In the rest of the paper, with a slight abuse of notation, $r_{i}(G)$ denotes $r_{i}(G, B)$ and $r_{i}(B)$ denotes $r_{i}(B, G){ }^{7}$

It is useful to define $P_{i}\left(\theta, \theta^{\prime}\right)$ as the (unconditional) probability of the event that type $\theta$ of candidate $i$ faces type $\theta^{\prime}$ of candidate $j$, and candidate $i$ uses the positive campaign. This unconditional probability is the sum of two terms: (i) the probability that type $\theta$ of player $i$ searches and uses a positive campaign, discovering the rival to be of type $\theta^{\prime}$, and (ii) the probability that type $\theta$ of player $i$ does not search and employs a positive campaign conditional on not searching.

$$
P_{i}\left(\theta, \theta^{\prime}\right)=p_{i}(\theta) r_{i}\left(\theta, \theta^{\prime}\right)+\left(1-p_{i}(\theta)\right) q_{i}(\theta), \text { where } \theta, \theta^{\prime}=G, B
$$

Expanding on Eq. (1), we define:

$$
\begin{aligned}
& P_{i}(B, G)=p_{i}(B) r_{i}(B)+\left(1-p_{i}(B)\right) q_{i}(B) \\
& P_{i}(G, B)=p_{i}(G) r_{i}(G)+\left(1-p_{i}(G)\right) q_{i}(G)
\end{aligned}
$$

Lemma 1, which deals with campaign choice for each type of candidate conditional on available information, brings out the hide-and-seek nature of the game.

Lemma 1 For $\theta \in\{G, B\}$ and $i=1,2$, the campaign choice $q_{i}(\theta)$ conditional on not searching, and the campaign choice $r_{i}(\theta)$ conditional on finding the rival type to be different from own type, are determined as follows:

(a) On finding the rival to be a bad type, the good type uses a positive campaign $\left(r_{i}(G)=1\right)$ if $P_{-i}(B, G)<\frac{1}{2}$ and a negative campaign $\left(r_{i}(G)=0\right)$ if $P_{-i}(B, G)>\frac{1}{2}$. Conditional on not searching, the good type employs a positive campaign $\left(q_{i}(G)=1\right)$ if $\operatorname{Pr}(B) \cdot P_{-i}(B, G)<\frac{1}{2}$ and a negative campaign $\left(q_{i}(G)=0\right)$ if $\operatorname{Pr}(B) \cdot P_{-i}(B, G)>\frac{1}{2}$, where $\operatorname{Pr}(B)=1-\alpha$.

(b) On finding the rival to be a good type, the bad type uses a positive campaign $\left(r_{i}(B)=1\right)$ if $P_{-i}(G, B)>\frac{1}{2}$ and a negative campaign $\left(r_{i}(B)=0\right)$ if $P_{-i}(G, B)<\frac{1}{2}$. Conditional on not searching, the bad type employs a positive campaign $\left(q_{i}(B)=1\right)$ if $\operatorname{Pr}(G) \cdot P_{-i}(G, B)>\frac{1}{2}$ and a negative campaign $\left(q_{i}(B)=0\right)$ if $\operatorname{Pr}(G) \cdot P_{-i}(G, B)<\frac{1}{2}$, where $\operatorname{Pr}(G)=\alpha$.

Proof See the Appendix.

According to Lemma 1, the campaign choice of a good type of player $i$ depends only on $P_{-i}(B, G)$, the probability with which she expects a bad type of the rival to employ the positive campaign against her. If the bad type goes positive with a high probability, the good type prefers to go negative and expose the bad type, and if the bad type goes

\footnotetext{
7 Thus, in the rest of the paper, $r_{i}(\theta)$ denotes the probability of type $\theta$ of player $i$ employing a positive campaign on discovering player $j$ to be of type $\{G, B\} \backslash \theta$.
} 
negative with a high probability, the good type prefers to go positive and reveal her own type. In the same way, the message of the bad type of player $i$ depends only on $P_{-i}(G, B)$, the probability that the good type of the rival uses a positive campaign against him. The bad type always prefers to avert a fruitful debate with the good type, and therefore tries to mimic the latter's campaign choice. As an implication of Lemma 1 part (a), conditional on not searching, the good type has a strictly dominant campaign choice $(P)$ if it is the more common type. Similarly, Lemma 1 part (b) implies that conditional on not searching, the bad type finds it strictly dominant to choose a negative campaign $(N)$ if the bad type is more common. In other words,

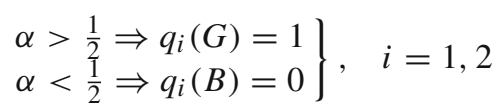

Proposition 1 demonstrates the equilibrium behavior of candidates. ${ }^{8}$

Proposition 1 The game admits a unique equilibrium. For $i=1$, 2, the equilibrium quantities for different values of the prior $\alpha$ are as follows:

(i) If $\alpha<c$, there is a fully separating equilibrium where no type searches, the good type uses the positive campaign and the bad type attacks, i.e., $p_{i}(G)=p_{i}(B)=$ $0, q_{i}(G)=1, q_{i}(B)=0$, and (off equilibrium), $r_{i}(G)=r_{i}(B)=1$.

(ii) If $\alpha \in(c, 1-c)$, there is a partially separating equilibrium where both types search with positive probability, i.e., $p_{i}(G)=\frac{1}{2}\left(1-\frac{c}{\alpha}\right), p_{i}(B)=\frac{1}{2}(1+$ $\left.\frac{c}{1-\alpha}\right)$.The good type employs a positive campaign when she does not search and a negative campaign when she searches and finds the rival to be a bad type, i.e., $q_{i}(G)=1$, and $r_{i}(G)=0$. The bad type employs a negative campaign when he does not search and a positive campaign when he searches and finds the rival to be a good type, i.e., $q_{i}(B)=0$ and $r_{i}(B)=1$.

(iii) If $\alpha>1-c$ there is a fully pooling equilibrium where both types send the positive campaign and neither type searches, i.e., $p_{i}(G)=p_{i}(B)=0, q_{i}(G)=$ $q_{i}(B)=1$, and (off equilibrium), $r_{i}(G)=0, r_{i}(B)=1$.

Proof In the appendix.

Notice that the unique equilibrium is type-symmetric in the sense that the same type of both players employ the same strategy in equilibrium. From now on, we shall drop the index $i$ from the relevant variables while discussing the players' strategies.

If either type is too rare (when $\alpha<c$ or $\alpha>1-c$ ), then there is no incentive for search, and each candidate acts as if the rival is of the common type. When $\alpha<c$, the bad type finds it strictly dominant to employ a negative campaign (by Eq. (3)). The action of the bad type is predictable, i.e., $P(B, G)=0$, and by Lemma 1 part (a), the good type employs a positive campaign. Thus, when expected candidate quality is very low, we have a "competitive" electorate, there is a lot of negative advertising, but the

\footnotetext{
8 We do not consider the case $\alpha=c$ since it is non-generic. In this case, we can have a continuum of equilibria. However, the equilibria discussed in the proposition extended to $c \rightarrow \alpha$ still exist in the limit $c=\alpha$. The case of $\alpha=1-c$ is not considered due to the same reasons.
} 
good type can always separate itself from the bad type by ensuring a fruitful debate. At the other extreme, when $\alpha>1-c$, the good type always goes positive (Eq. (3)), and Lemma 1 part (b) dictates that then the bad type will use a positive campaign too, and successfully ensure cross talk. Therefore, when the expected candidate quality is very high, we have a "conservative" electorate, where there is only positive advertising, and it is impossible to distinguish the good type from the bad. Notice that the no-search case demonstrates that the rarer type has the advantage in the hide-and-seek game. ${ }^{9}$

Search is undertaken only when neither type is very rare, i.e., the expected candidate quality is moderate $(c<\alpha<1-c)$. The good candidate always provides arguments supporting himself (positive campaign) unless he is sure that the rival is a bad type, in which case he tries to expose the rival by going negative. The bad candidate on the other hand has a default campaign which is negative, but when he is sure that the rival is a good type, he tries to ensure cross talk by defending himself (positive campaign), hoping that the rival has not searched and is going to employ a positive campaign too. This equilibrium is supported by the fact that the good candidate searches less frequently than the bad type.

To see the technical intuition for the equilibrium with search, notice that if a candidate were to engage in costly search with any positive probability, he must play different actions with different types of the rival. Since the good type plays $P$ if the rival is also good, he must play $N$ when search reveals the rival to be bad, i.e., $r(G)=0$. In the hide-and-seek game with the bad type, the good type does not want to play the same action against the bad type all the time. Hence, the good type uses $P$ conditional on not searching and $N$ conditional on searching and discovering the rival to be bad. Similarly, the bad type has $r(B)=1$ and $q(B)=0$. The search probabilities are chosen by each type so as to keep the "other" type indifferent between searching and not searching: Search thus performs the role of mixing between the two different actions in the hide-and-seek game.

As demonstrated by the above proposition, irrespective of whether search occurs or not, when two good candidates are in competition, there is cross talk with both candidates arguing in support of themselves (positive campaign). When two bad candidates face each other, we again have cross talk, but with negative campaigns if $\alpha<1-c$ and with positive campaigns otherwise. A fruitful debate can occur only between a good and a bad type.

Although we have considered "mechanistic" or passive voting, including the voter as a rational player does not alter this equilibrium. First, note there is nothing more to learn from cross-talk by way of Bayesian inference because both candidates take the same action. As long as there is a common prior over both candidates, it is rational for the voter to randomly choose the winner. In equilibrium, fruitful debate occurs only between two different types of candidates - thus the passive action is again rational. We make the additional assumption that off the equilibrium, if the voter observes a candidate to be of a type that is not supposed to be observed in equilibrium (e.g., type $B$ in the case $\alpha<c$ ), then the voter assumes that each type of the rival candidate

9 If $c>\frac{1}{2}$, then we have either $\alpha<c$ or $1-\alpha<c$, and in the same way as proposition 1(i) and 1(iii), there is no search. For $\alpha<\frac{1}{2}$, the equilibrium is fully separating with the good type going positive and the bad type going negative; and for $\alpha>\frac{1}{2}$, the equilibrium is fully pooling with both types going positive. 
has a small (not necessarily equal) positive probability of having played $N$, which implies that there remains an uncertainty about the type of the rival. Thus, off the equilibrium path too, the voter strictly prefers to vote for the candidate revealed to be good and against the candidate revealed to be bad. Therefore, the "naive" voting action hardwired in the payoffs does not change if we include a rational voter in the model. Voter inference is formally discussed in the online appendix.

\subsection{Properties of search}

A few properties of search are worth mentioning here:

1. Given the equilibrium campaign strategies, search has the property of strategic substitutability: Certain search by one type takes away the incentive of the other type to search. Moreover, the type that does not search can mix messages in such a way as to nullify the informational advantage of the type that has searched. Therefore, no candidate searches with certainty, even if search cost is very low. The result that search must be probabilistic casts doubt over the assumption of full information between candidates which is assumed in PY. ${ }^{10}$

2. Search is reciprocal, i.e., if one candidate searches with a positive probability, the other does so too.

3. If search occurs, the bad type searches with a higher frequency than the good type. In the equilibrium with search, we must have $p(B)>\frac{1}{2}>p(G)$. Notice that the ex-ante marginal value of positive advertising is higher to the good type than the bad. It is this advantage that depresses the good type's incentive to search, and raises the bad type's motivation for the same. The cost of search drives a wedge between the incentives to search of the two types: For any given $\alpha$ for which search is worthwhile in equilibrium, $p(B)-\frac{1}{2}$ and $\frac{1}{2}-p(G)$ are both increasing in $c$ : This leads to an inefficiency which we discuss later in the discussion on candidate selection. To drive the point home further, note that as $c \rightarrow 0$, both $p(B)$ and $p(G)$ converge to $\frac{1}{2}$ and the inefficiency due to search vanishes in the limit.

\subsection{Campaigns and candidate quality}

There is a large empirical/experimental literature discussing the effect of negative advertising. The main finding is that attack advertising hurts both the sponsor and the target of the negative campaign, although there is considerable disagreement on the relative and absolute size of this effect. Recent formal work by Mattes (2007) has provided a theoretical foundation for these effects by studying voter inference of candidate qualities from their campaign strategies. The most important issue highlighted in the current paper is that information about candidate quality is revealed by the profile of campaign choices rather than the choice by an individual candidate: A negative

10 The result that search is always probabilistic depends on the assumption that search is private. In a model where each candidate knows whether the rival has searched or not and can make their campaign choice conditional on such information, search is never probabilistic: for all parameter values in a type-symmetric equilibrium, a given type either serches or does not search. Mixing happens at the level of campaign choice. 
campaign may have very different effects depending on whether it is met by a positive or a negative campaign.

\subsubsection{Campaign profiles and information revelation}

The profile of campaigns reveal information to the voter directly through the information revelation protocol, and additionally through equilibrium inferences. To analyze voter inference, one really has to look at the voter as a rational player. We have informally discussed earlier (and formally demonstrated in the online appendix) that the equilibrium strategies derived in this section can be used to analyze voter inference of candidate types from campaign choices.

A fruitful debate reveals the true quality of the focal candidate. Moreover, the rival candidate's type can also be inferred with certainty by the rational voters. In equilibrium, a fruitful debate can occur only between opposite types: Such a debate has either a good candidate "exposing" a bad one, or a good candidate successfully defending herself against attacks by a bad one.

In case of cross talk, there is no new information revealed directly through debate. In equilibrium, voters cannot distinguish between the two candidates as they are observed to take the same action. However, we can talk about the effect of campaign choice in terms of whether the prior, i.e., expected candidate quality is updated upwards or downwards. When there is cross talk with positive campaigns, either or both of the candidates must be good. On the other hand, when both candidates use negative campaigns, at least one of the two candidates must be bad, and voters adjust their assessment downwards. This feature of equilibrium leads to voter inferences under cross talk discussed below.

Formally, suppose both candidates choose the same campaign $M_{1}=M_{2}=M \in$ $\{P, N\}$, and the inferred probability of the candidates being good is $\widehat{\alpha}(M)$. Then,

1. If $\alpha \in(c, 1-c)$, then $\widehat{\alpha}(N)<\min \left\{\alpha, \frac{1}{2}\right\}$ i.e., when both candidates employ negative campaigns, the assessment of candidate quality goes down. In fact, irrespective of the prior $\alpha$, the voters believe that both the candidates are more likely to be bad than good. The opposite conclusions hold when each candidate runs a positive campaign, i.e., $\widehat{\alpha}(P)>\max \left\{\alpha, \frac{1}{2}\right\}$.

2. If $\alpha>1-c$ there is full pooling: The campaign choice is completely uninformative about quality, and thus $\widehat{\alpha}(P)=\alpha$.

3. If $\alpha<c$, we have full separation. Then, anyone using a positive campaign distinguishes himself as a good type while anyone attacking the rival in debate reveals himself to be a bad type. Formally, in this case, $\widehat{\alpha}(N)=0$.

\subsubsection{Effect of negative advertising}

In order to compare our results directly with the existing literature on negative campaigns, we now turn to the following question: Suppose a candidate is observed to run a negative campaign. What effect does our model predict about the sponsor and the target? The answer, in our model, depends on what campaign the target is observed to run. If the rival also goes negative, the expected quality of both the target and the 
sponsor goes down. On the other hand, if the rival runs a positive campaign, the quality assessment of the sponsor of the negative campaign and that of the target move in opposite directions.

For the case of a fruitful debate (unmatched messages), there are two cases to consider. When $\alpha<c$, only the bad type runs a negative campaign: Thus the sponsor of a negative campaign is hurt and the target benefits. When $\alpha \in(c, 1-c)$, the effect is ambiguous since both good and bad types run negative campaigns. In particular, the attacker in a fruitful debate is revealed to be good with probability $t(\alpha)$, where

$$
t(\alpha)=\frac{p(G) p(B)}{p(G) p(B)+(1-p(G))(1-p(B))},
$$

where $p(G)$ and $p(B)$ follow from Proposition 1 (ii). It can be checked that $t(\alpha)>\frac{1}{2}$ if and only if $\alpha>\frac{1}{2}$. Remark 1 sums up the effect of a negative campaign when the rival uses a positive campaign in equilibrium.

Remark 1 Suppose in equilibrium, one candidate runs a negative campaign and the rival runs a positive campaign. The negative campaigner is more likely to win than lose (and the positive campaigner is more likely to lose than win) if and only if the bad type is rarer, i.e., $\alpha>\frac{1}{2}$.

Later, we observe in Remark 2 that when $\alpha<\frac{1}{2}$, a random candidate is more likely to run a negative campaign and when $\alpha>\frac{1}{2}$, he is more likely to run a positive campaign. Therefore, when a negative campaign is faced with a positive one, the attacker has the advantage if most candidates are expected to run positive campaigns and the attacker has a disadvantage if most candidates are expected to go negative. In other words, the candidate using the less expected mode of campaign has the advantage. PY (Proposition 4) has the opposite finding: When two candidates choose "unmatched" messages, the one using the dominant mode of campaigning is at an advantage. The main reason for the difference is that while PY consider only informative campaigns, we consider softer information that leads to a hide-and-seek framework.

\section{Comparative statics}

In this section, we examine the comparative static properties of the equilibrium for different levels of the search cost and average candidate quality, and discuss the implications of such properties.

\subsection{Welfare analysis: candidate selection}

Looking at political campaigns as debates between candidates with partial information about each other helps us understand a few important issues about the efficiency of the campaign process especially in terms of its ability to select the better candidate. The major finding of the model is that as the prior probability of a candidate being good increases, the probability of a good candidate being selected through the electoral process may actually go down. Figure 1 plots the total probability of selection of 
Fig. 1 Total probability of selection of a good candidate

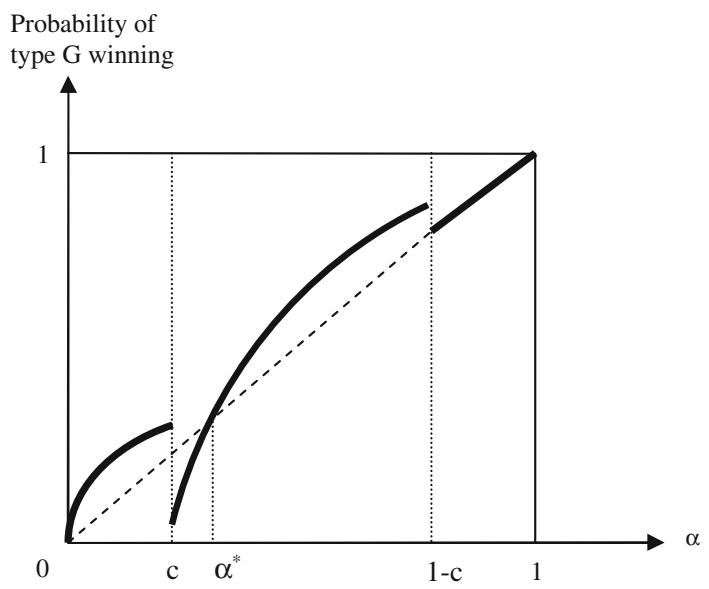

the good candidate against the prior $\alpha$ for some given search cost $c .{ }^{11}$ In the graph, there exist downward jumps in otherwise piecewise continuous and monotonically increasing graphs. There are three regimes based on ranges of $\alpha$ : Full separation, partial separation and full pooling, and the downward jumps occur when we move from a more efficient regime to a less efficient one as the average candidate quality increases. Note also that since an increase in the search cost favours the bad type, the downward jumps are larger as the cost of information increases.

The total probability of selection may not fully reflect the efficiency of debate as a selection mechanism. Perhaps a better indicator of the efficiency of debates in selecting the right candidate would be a measure of how often the good candidate wins when competing against a bad candidate. Hence we look at the equilibrium probability of a fruitful debate conditional on candidates being of different types. Denote this probability $\beta(\alpha, c)$. Proposition 2 shows how $\beta$ changes with the parameters of the model.

Proposition 2 Suppose one of the two competing candidates is a good type and the other is a bad type. Debate is always fruitful if $\alpha<c$, never fruitful if $\alpha>1-c$, and if $\alpha \in(c, 1-c)$ debate is fruitful with a probability $\beta(\alpha, c)=\frac{1}{2}-\frac{c^{2}}{2 \alpha(1-\alpha)}$.

Proof Follows from Proposition 1.

When there is no search, the rarer type has full advantage in the hide-and-seek game. For very low priors, debate is fully efficient and for very high priors, debate is fully inefficient. When the prior is moderate, both types search in equilibrium.

In the case when the search cost is very low, i.e., $c \rightarrow 0$, we have $p(B)$ and $p(G)$ both converging to $\frac{1}{2}$. In this case, the debate between the good and bad types reduces to the matching pennies game in which there is no advantage to either type: Hence each message profile occurs with equal probability. Then $\beta(\alpha, c) \rightarrow \frac{1}{2}$ for almost all

${ }^{11}$ Denote the total probability of selection of the good type given $c$ and $\alpha$ as $f_{c}(\alpha)$. It can be deduced from Proposition 1 that: $f_{c}(\alpha)=\left\{\begin{array}{l}2 \alpha-\alpha^{2} \text { if } \alpha<c \\ \frac{3}{2} \alpha-\frac{1}{2} \alpha^{2}-\frac{1}{2} c^{2} \text { if } c<\alpha<1-c \\ \alpha \text { if } \alpha>1-c\end{array}\right.$ 
Fig. 2 Probability of correct selection when the two candidates are of different types

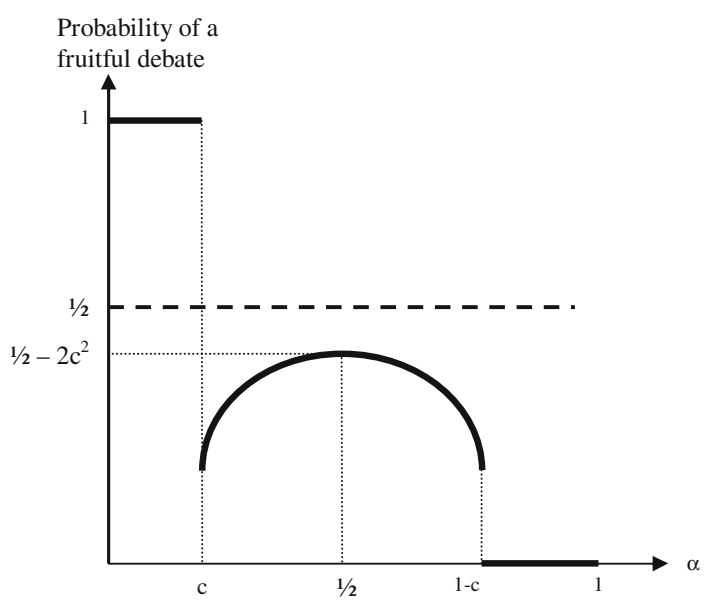

values of the prior. As the search cost increases, there is a further inefficiency due to differential incentives for search that the two types have. As mentioned before, we have $p(G)<\frac{1}{2}<p(B)$; moreover, both $\left|p(G)-\frac{1}{2}\right|$ and $\left|p(B)-\frac{1}{2}\right|$ are increasing in $c$. This inefficiency due to differential incentives for search leads to a further welfare loss of $\frac{c^{2}}{2 \alpha(1-\alpha)}$. Figure 2 shows how $\beta(\alpha, c)$ changes for different values of $\alpha$. The bold dashed line shows $\beta$ when $c \rightarrow 0$.

Given an expected candidate quality $\alpha$, if a social planner could choose the search cost $c$ (or more realistically, the extent of office perks), what would the best choice be? The answer depends on whether the good or the bad type is more common.

If $\alpha>\frac{1}{2}$, we unambiguously improve welfare by reducing $c$, and the best choice of $c$ would be as close to zero as possible. On the other hand, if the bad type is more common, there are two opposing effects. When $c<\alpha$, a marginal reduction in $c$ reduces the inefficiency due to search and increases $\beta$. On the other hand, for any given $\alpha$ less than $\frac{1}{2}$, if we set $c>\alpha$, we get full separation of types. Hence, if the bad type is more common, a social planner can achieve full separation by sufficiently reducing the prize from office.

\subsection{Negative advertising}

Next, we look at the type of messages exchanged in the debate as the prior varies. As the average candidate quality improves, the ex-ante probability that a candidate will employ a negative campaign goes down. In general, the bad type's propensity to attack goes down as the probability of the rival being good goes up. For moderate values of the prior, the propensity of negative campaigning decreases with $\alpha$ because the good type's increasing aggressiveness (in searching and attacking the bad type) is more than compensated by the bad type's increasing conservatism (in searching and sending positive messages). This is illustrated in Fig. 3 and stated in Remark 2, which follows from Proposition 1. ${ }^{12}$

12 The probability of a negative message $\gamma(\alpha, c)$ is: $\gamma(\alpha, c)=\left\{\begin{array}{l}1-\alpha \text { if } \alpha<c \\ (1-\alpha)-\frac{c}{2}(1-2 \alpha) \text { if } \alpha \in(c, 1-c) \\ 0 \text { if } \alpha>1-c\end{array}\right.$ 
Fig. 3 Probability of a negative campaign as a function of the prior

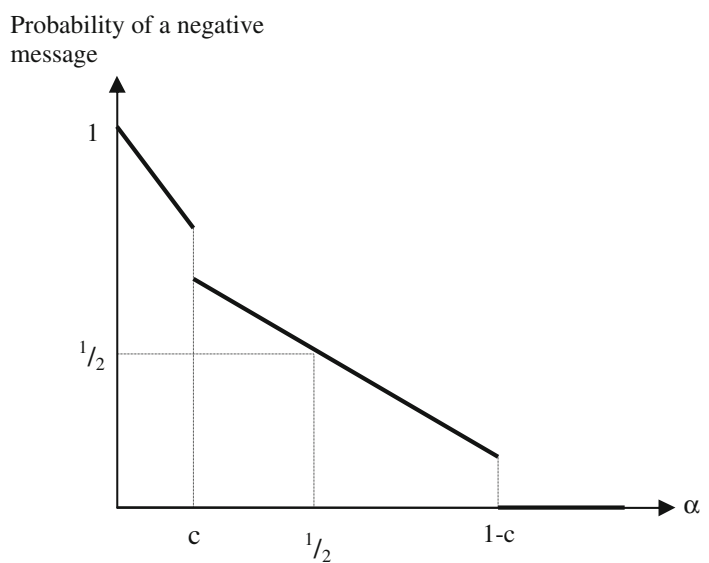

Remark 2 The probability of negative advertising is strictly decreasing in the prior for $\alpha<1-c$ and is equal to 0 for $\alpha>1-c$. Negative advertising is more frequent than positive advertising if the bad type is more common, and less frequent if the good type is more common.

At this stage, one may want to distinguish between a negative campaign targeted against a bad type from an attack on a good type, presumably because the latter is likely to be less truthful than the former. In this model, a good candidate never wages a negative campaign against a good candidate. A good candidate is subjected to a negative campaign only by a bad candidate that has not searched.

Just like negative advertising as a whole, the incidence of negative advertising targeted against a good type goes down as the prior improves. ${ }^{13}$ The probability of attacks on the good type decreases with improvement in expected candidate quality because the bad type becomes less common and also starts searching more often. As the expected candidate quality improves, a growing share of negative advertising is informed attacks by the good type. In this sense, the share of truth in negative campaigns goes up as the expected candidate quality improves. Therefore, we have a "cleaner" electorate as the prior increases. However, this also implies that there is less information revelation through debate and as shown before, the selection performance worsens.

\section{Extensions}

The hide-and-seek framework can be extended to discuss several features necessary for a richer model of politics.

\footnotetext{
13 The probability of attack on a good type is $1-\alpha$ for $\alpha<c, \frac{1}{2}(1-\alpha)-\frac{c}{2}$ if $\alpha \in(c, 1-c)$ and 0 if $\alpha>1-c$.
} 


\subsection{Probabilistic information revelation}

Consider a generalization of the model discussed in Sects. 2 and 3 where the quality of the candidate is revealed to the voter with a probability dependent on the profile of campaigns employed. Suppose the revelation of a candidate's quality follows a Bernoulli random variable distributed independently conditional on the campaign profile. If both candidates use positive advertising, each candidate's quality is revealed to the voter independently with probability $\phi_{P}$, and similarly, with probability $\phi_{N}$ if both engage in negative advertising. If, on the other hand, one candidate employs a positive campaign and the other a negative campaign, then the focal candidate's type is revealed with probability $\pi_{P}$ and the non-focal candidate's type with probability $\pi_{N}$, again, independently of each other. We call this structure the "probabilistic information revelation protocol". We make two assumptions on the parameters:

$$
\begin{aligned}
& \text { (A1) } 1 \geq \pi_{P}>\pi_{N} \geq 0 \\
& \text { (A2) }\left(1-\pi_{P}\right)\left(1-\pi_{N}\right)<\min \left\{\left(1-\phi_{P}\right)^{2},\left(1-\phi_{N}\right)^{2}\right\}
\end{aligned}
$$

(A1) captures the fact that in a fruitful debate, the quality of a focal candidate is revealed with a higher probability than that of the non-focal candidate, and (A2) says that the probability that the voter learns nothing is lower in a fruitful debate than in cross talk. ${ }^{14}$ First, we demonstrate that under a probabilistic information revelation protocol, these two assumptions preserve the incentive structure in the basic framework.

If the types of both candidates are revealed, the voter compares the two and decides. If only one type is revealed, the voter votes for the candidate if he is revealed to be good and against him if he is revealed to be bad. If neither type is revealed, the voter votes randomly. In the notation used in Sect. 2, the payoff to the row player from each campaign profile when the two candidates are of the same type are as follows:

\begin{tabular}{|c|c|c|}
\multicolumn{3}{c}{ G-type } \\
\hline & $\mathrm{P}$ & $\mathrm{N}$ \\
\hline $\mathrm{P}$ & 1 & $k$ \\
\cline { 2 - 3 } $\mathrm{N}$ & $(2-k)$ & 1 \\
\hline
\end{tabular}

B-type \begin{tabular}{|c|c|c|}
\multicolumn{3}{c}{ B-type } \\
\hline $\mathrm{P}$ & $\mathrm{P}$ & $\mathrm{N}$ \\
\cline { 2 - 3 } $\mathrm{N}$ & $(2-m)$ & 1 \\
\hline
\end{tabular}

\footnotetext{
14 Notice that even if we let $\pi_{N}=0$, a simple comparison of $\pi_{P}$ and $\phi_{P}$ (or $\phi_{N}$ ) will not allow us to compare the amount of information revealed in a fruitful debate with that in cross talk. The reason is that while in a cross talk, each candidate's quality is revealed with probability $\phi_{P}$ (or $\phi_{N}$ ) independently, $\pi_{P}$ is the revelation probability only for the focal candidate.
} 
where $k=\pi_{P} \pi_{N}+\left(1-\pi_{P}\right)\left(1-\pi_{N}\right)+2 \pi_{P}\left(1-\pi_{N}\right)=1+\left(\pi_{P}-\pi_{N}\right)$, and similarly, $m=1-\left(\pi_{P}-\pi_{N}\right)$.

Notice that assumption ( $A 1$ ) implies (and is implied by) $1<k \leq 2$ and $0 \leq m<1$. This ensures that when a good type meets another good type, both find it strictly dominant to use positive advertising; and when a bad type meets another bad type, both find it strictly dominant to use negative campaigning. Next, consider the game between a good type and a bad type, where we can find the row player's payoffs in the individual cells through a little algebra.

\begin{tabular}{|c|c|c|}
\multicolumn{3}{c}{ B-type } \\
\hline & $\mathrm{P}$ & $\mathrm{N}$ \\
\hline $\mathrm{P}$ & $a_{1}$ & $b$ \\
\cline { 2 - 3 } $\mathrm{N}$ & $b$ & $a_{2}$ \\
\hline
\end{tabular}

$$
\text { where }\left\{\begin{aligned}
a_{1} & =2-\left(1-\phi_{P}\right)^{2} \\
a_{2} & =2-\left(1-\phi_{N}\right)^{2} \\
b & =2-\left(1-\pi_{P}\right)\left(1-\pi_{N}\right)
\end{aligned}\right.
$$

Assumption (A2) is equivalent to $b>\max \left(a_{1}, a_{2}\right)$, which induces the matching pennies structure. Therefore, when a good type faces a bad type, the former prefers fruitful debate (unmatched messages) and the latter prefers cross talk (matched messages).

The following proposition describes the equilibrium in the probabilistic revelation case. Under two additional restrictions, the features of the equilibrium are exactly the same as those in the deterministic revelation case. Denoting $\pi_{p}-\pi_{N}$ by $\pi$, we need (i) $c<\frac{\left(b-a_{1}\right)^{2}}{\left(b-a_{1}\right)+\pi}$ and (ii) $b-a_{1}<\pi$. The first restriction simply requires the cost of information acquisition to be small enough, and the second one requires that under fruitful debate, the probability of the revelation of the focal candidate's type is sufficiently higher than the probability of revelation of the non-focal candidate's type. The second restriction is always satisfied if we assume that $\pi_{N}=0$, i.e., a fruitful debate reveals information about the focal candidate only, and not about the non-focal candidate. With these two restrictions, Proposition 1 is a special case of Proposition 3.

Proposition 3 Assume (A1), (A2), $c<\frac{\left(b-a_{1}\right)^{2}}{\left(b-a_{1}\right)+\pi}$ and $b-a_{1}<\pi$. The following is the unique equilibrium for different values of the prior $\alpha$ :

(i) If $\alpha<\min \left(\frac{c}{b-a_{1}}, \frac{1}{2}\right)$ there is a fully separating equilibrium with no search, i.e., $p_{i}(G)=p_{i}(B)=0, q_{i}(G)=1, q_{i}(B)=0$, and (off equilibrium), $r_{i}(G)=r_{i}(B)=1$.

(ii) If $\frac{c}{b-a_{1}}<\frac{1}{2}$, then for $\alpha \in\left(\frac{c}{b-a_{1}}, 1-\frac{c}{b-a_{1}}\right)$, there is a partially separating equilibrium where both types search with positive probability, and $q_{i}(G)=1$, $r_{i}(G)=0, q_{i}(B)=0, r_{i}(B)=1, p_{i}(G)=\frac{1}{2 b-\left(a_{1}+a_{2}\right)}\left[\left(b-a_{1}\right)-\frac{c}{\alpha}\right]$, and $p_{i}(B)=\frac{1}{2 b-\left(a_{1}+a_{2}\right)}\left[\left(b-a_{2}\right)+\frac{c}{1-\alpha}\right]$. 
(iii) If $\alpha>\max \left(1-\frac{c}{b-a_{1}}, \frac{1}{2}\right)$ there is a fully pooling equilibrium on the positive message and neither type searches, i.e., $p_{i}(G)=p_{i}(B)=0, q_{i}(G)=q_{i}(B)=$ 1 , and (off equilibrium), $r_{i}(G)=0, r_{i}(B)=1$.

The proof of the proposition is provided in the online appendix as it is similar to the proof of Proposition 1. We note here that for the probabilistic information revelation model, the assumption of "passive" voting is no longer innocuous: We need to assume that voter inference is based only on the arguments made in the campaign and not additionally on guessing the strategic motivations of candidates for making particular campaign choices.

\subsection{Continuous type space}

With the deterministic information revelation protocol, we have a natural extension of our model to the continuous type case. To discuss the continuous type extension we need to formally include the voter as a third player in the game and discuss her beliefs given candidate strategies and revealed information. In the online appendix, we discuss voter beliefs and then show that there exists a class of type-symmetric equilibria of the following kind: There is a cut-off quality $\theta^{*}$ such that candidates with their type above the cut-off behave like the good type in the discrete model, and those with type below the cut-off behave like the bad type. There are multiple equilibria, with $\theta^{*}$ taking any value in an interval. In this class of equilibria, all results in the discrete model carry over. Moreover, we get an endogenous classification of the continuum of quality into "good" and "bad", dictated by voter beliefs.

The continuous type extension is not of mere technical interest. It provides a comparison with the existing literature on elections as adverse selection mechanisms with one principal (the median voter) and two competing agents (the candidates). Banks (1990) analyzes Downsian competition where candidates may make false announcements about their preferred positions, but lying has an exogenous cost that increases in the distance between their preferred and announced positions. While Banks finds pooling of candidate types over an interval containing the median voter's most preferred position, Callander and Wilkie (2007) shows that if there is a cheap talking type in the model, pooling happens at two disjoint intervals on either side of the median voter's ideal point. Thus, the "best" types (those that are preferred most by the median voter) pool in the former paper while the "moderate" types pool in the latter, and all the other types separate. The continuous type space in this model can be interpreted as a space of possible candidate locations on the Hotelling line, with higher quality implying a location closer to the median voter's ideal position. In the equilibrium in the current model there are two clusters of pooling-one for the "good" types and the other for the "bad" types. The message in this paper is that if competition reveals information about only one candidate, it is possible to separate the good set of types from the bad set of types (where good and bad types are defined endogenously), but one cannot separate within the good or bad set of types.

It is important to note that there are multiple type-symmetric equilibria in the continuous type model. We use two refinement criteria: (i) better types must obtain 
weakly higher expected payoff in equilibrium and (ii) all types are revealed with positive probability in equilibrium. The first criterion is also used in PY, in a stronger form. The second criterion ensures that off-equilibrium beliefs about types play no role in equilibrium behavior. The class of equilibria we identify is the only one satisfying both these refinements and an additional technical restriction.

\subsection{Asymmetric strategies}

The payoff benchmark game described in Sect. 2 has a unique equilibrium where candidates play type-symmetric strategies. This is an artifact of an assumption embedded in the payoff structure: The voter votes for each candidate with equal probability when she is indifferent. In this equilibrium, focality arises only due to the information revelation protocol. However, once we include the voter as a rational player (as in the game discussed in the online appendix), there could be other equilibria. In these equilibria, the voter treats the two candidates asymmetrically, i.e., votes for one candidate or the other when indifferent. Here, focality arises additionally due to players co-ordinating on one strategy profile or another. For instance, there exists one equilibrium where neither candidate searches, candidate 1 uses a positive message irrespective of his type and candidate 2 employs a negative campaign irrespective of his type. This is supported by the off-equilibrium belief that the voter will vote for candidate 1 if she observes cross-talk with positive messages, and that she will vote for candidate 2 if both employ negative messages. ${ }^{15}$

\subsection{Uninformative campaigns}

In the paper, we have only allowed a candidate the choice to run a campaign about one of two issues: Own quality (positive campaign) or the rival's quality (negative campaign). Information is revealed if and only if both candidates focus on the same issue. One might legitimately ask whether our results would change if a candidate could run a "null campaign" in which he makes statements that are informative about neither candidate's quality irrespective of whether it is faced with a positive or a negative campaign. ${ }^{16}$ In other words, a "null campaign" would allow a candidate to ensure cross-talk unilaterally. The details of the analysis depends on how we model the payoffs, but the broad message is that it is not in the interest of the good type to use null campaigns.

First, suppose we extend the benchmark model (with mechanistic voting) presented in Sect. 2 to include null campaigns. An obvious starting assumption is that if either candidate employs a null campaign $\left(M_{i}=\phi\right)$, then no information is revealed to the voter about candidate quality. Under mechanistic voting, the debate payoff is shared equally between the two candidates. The payoff to the row player is presented as follows.

\footnotetext{
15 We thank an anonymous referee for suggesting this line of enquiry.

16 We thank an anonymous referee for suggesting this extension.
} 


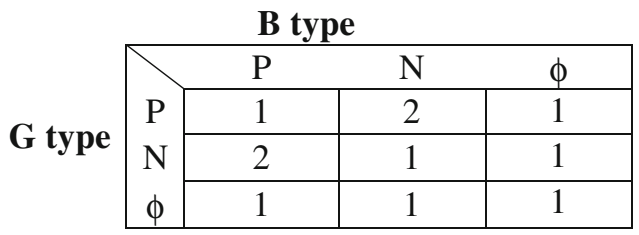

\begin{tabular}{c}
\multicolumn{5}{c}{ G type } \\
\hline
\end{tabular}

B type \begin{tabular}{|c|c|c|c|}
\hline & $\mathrm{P}$ & $\mathrm{N}$ & $\phi$ \\
\hline $\mathrm{P}$ & 1 & 0 & 1 \\
\cline { 2 - 4 } $\mathrm{N}$ & 2 & 1 & 1 \\
\cline { 2 - 4 }$\phi$ & 1 & 1 & 1 \\
\hline
\end{tabular}

Payoffs with uninformative messages (mechanistic voting)

In this game, for the good type, a positive campaign cannot do any worse than a null campaign irrespective of what the other candidate does. Therefore, in any equilibrium where a candidate does not employ a weakly dominated action, a good type will never employ a null campaign. In equilibrium of this mechanistic voting game, the only type to ever run a null campaign will be the bad type.

Next, we take a step further and look at the game with a rational voter. In this case, even if no information is directly revealed by the message profile, the voter can update her belief about the type of the candidate from the equilibrium strategies given the message profile. The equilibrium in the rational voter game that mirrors the logic presented in the previous paragraph is simply the one which replicates the outcome of the binary message game identified in Proposition 1. In this equilibrium, the voter rationally believes that only the bad type has an incentive to play the null message. Therefore, whenever a null message is employed against a non-null one, the candidate using the null message loses the debate. The payoff structure in the rational voter game induced by this belief is given below. 


\begin{tabular}{|c|c|c|c|c|}
\hline \multicolumn{5}{|c|}{ B type } \\
\hline \multirow{4}{*}{ G type } & & $\mathrm{P}$ & $\mathrm{N}$ & $\phi$ \\
\hline & $\mathrm{P}$ & 1 & 2 & 1 \\
\hline & $\mathrm{N}$ & 2 & 1 & 1 \\
\hline & $\phi$ & 1 & 1 & 1 \\
\hline
\end{tabular}

G type \begin{tabular}{c|c|c|c|}
\hline & $\mathrm{P}$ & $\mathrm{N}$ & $\phi$ \\
\hline $\mathrm{P}$ & 1 & 2 & 2 \\
\cline { 3 - 5 } & 0 & 1 & 2 \\
\cline { 3 - 4 } & 0 & 0 & 1 \\
\hline
\end{tabular}

\begin{tabular}{|c|c|c|c|c|}
\hline \multicolumn{5}{|c|}{ B type } \\
\hline \multirow{4}{*}{ B type } & & $\mathrm{P}$ & $\mathrm{N}$ & $\phi$ \\
\hline & $P$ & 1 & 0 & 2 \\
\hline & $\mathrm{N}$ & 2 & 1 & 2 \\
\hline & $\phi$ & 0 & 0 & 1 \\
\hline
\end{tabular}

Payoffs with uninformative messages (rational voting)

It is easy to check that given this payoff structure, the equilibrium outcome in Proposition 1 remains unaltered. For an easy intuition, notice that in the normal form, the null message is weakly dominated for the good type. Once the null message is eliminated for the good type, the null message is weakly dominated for the bad type too. Therefore, we have an equilibrium outcome where the good type finds the null message useless, and the bad type avoids the null message in order that he is not "outed" with certainty. Our result implies that while candidates may often use uninformative messages, a whole campaign that is composed only of uninformative statements may not be observed in the real world.

\section{Conclusion}

This paper examines the choice between positive and negative campaigns as a particular case of the more general decision problem of contradicting the opponent's argument (revealing information through fruitful debate) and raising a different subject (concealing information through cross talk). The fundamental idea of the paper is that information transmitted in a debate is conditionally verifiable: The truth of an argument is more transparent when compared with a counter-argument. Previous 
formal theoretic work on positive vs. negative advertising has considered only truthful statements in advertising. Our model incorporates softer information as part of possible messages spoken in advertising campaigns. The model points out that a lie or ad hominem attack, while useless to the voter, might be useful to the candidate in "muddling" the debate and thus distracting the attention of the electorate. The model also shows that, contrary to popular perception, voter welfare is not monotonic in the expected candidate quality.

The existing body of empirical and experimental work investigating the role of attack messages has largely proved inconclusive in establishing whether such messages are beneficial or harmful. The current paper suggests that perhaps efforts to classify negative campaigns in such exclusive categories might be misdirected. We present a more nuanced picture in which negative campaigns may sometimes be useful and sometimes not-so-useful to the voter, depending on the motivation of the candidate running the campaign. We point out the information is often revealed jointly by the two candidates discussing two sides of the same issue - and for future work, one might need to take into account the entire profile of messages in order to set up the right hypotheses to test with field or laboratory data.

Open Access This article is distributed under the terms of the Creative Commons Attribution 4.0 International License (http://creativecommons.org/licenses/by/4.0/), which permits unrestricted use, distribution, and reproduction in any medium, provided you give appropriate credit to the original author(s) and the source, provide a link to the Creative Commons license, and indicate if changes were made.

\section{Appendix: Proof of Proposition 1}

To define the equilibrium strategies in this setting, some basic notation needs to be introduced.

Denote a pure strategy for player $i$ by $s_{i}=(p(\cdot), q(\cdot)$ and $r(\cdot, \cdot)), i=1,2$. Next, define $E u_{i}^{\theta}\left(M \mid s_{-i}, I\right)$ as the expected utility from debate to type $\theta \in\{G, B\}$ of player $i$ from playing message $M \in\{P, N\}$ when player $-i$ is using strategies $s_{-i}$, and the information available to player $i$ is $I \in\{G, B, \phi\}$. If there is search and the type of the rival $\left(\theta_{-i}\right)$ is known by $i$ then $I=G$ or $I=B$, else, $I=\phi$. This expected utility is constructed from the debate payoff $u_{i}(\mathbf{M}, \theta)$, taking expectation over the possible messages of the rival $-i$ and if the rival type is not known, then over possible types of the rival too. Define as $E u_{i}^{\theta}\left(m \mid s_{-i}, I\right)$ the expected utility when type $\theta$ of player $i$ plays message $P$ with a probability $m \in[0,1]$.

Define $E U_{i}^{\theta}\left(S \mid s_{-i}\right)=E_{\left(\theta^{\prime}\right)}\left[\underset{m \in[0,1]}{\arg \max } E u_{i}^{\theta}\left(m \mid s_{-i}, \theta^{\prime}\right)\right]-c$ as the expected utility from search, taking into account the optimal message choice post search, and taking expectation over rival types and $E U_{i}^{\theta}\left(N S, m \mid s_{-i}\right)=E u_{i}^{\theta}\left(m \mid s_{-i}, \phi\right)$ as the expected utility from not searching and playing a mix $m$ of messages.

When a candidate of type $\theta$ knows that his rival is also of type $\theta$, then the candidate has a strictly dominant message. Denote this message by $D(\theta)$. In other words, $D(G)=P$ and $D(B)=N$. Denote by $\overline{D(\theta)}$ the other available action in the message 
space, i.e. $\overline{D(\theta)}=\{P, N\} \backslash D(\theta)$. In the same way, denote by $\bar{\theta}$ the type different from $\theta$, i.e., $\bar{\theta}=\{G, B\} \backslash \theta$.

We shall first prove a few results in the form of claims and use those results to find the equilibrium strategies for different parameter values.

Claim $1 E u_{i}^{\theta}\left(D(\theta) \mid s_{-i}, \theta\right)-E u_{i}^{\theta}\left(\overline{D(\theta)} \mid s_{-i}, \theta\right)=1$

Proof For $\theta=G, D(\theta)=P$, and $\overline{D(\theta)}=N$

$E u_{i}^{G}\left(P \mid s_{-i}, G\right)-E u_{i}^{G}\left(N \mid s_{-i}, G\right)=\left\{P_{-i}(G, G)+2\left(1-P_{-i}(G, G)\right)\right\}-$ $\left\{\left(1-P_{-i}(G, G)\right\}=1\right.$

For $\theta=B, D(\theta)=N$, and $\overline{D(\theta)}=P$

$E u_{i}^{B}\left(N \mid s_{-i}, B\right)-E u_{i}^{B}\left(P \mid s_{-i}, B\right)=\left\{\left(1-P_{-i}(B, B)\right)+2 P_{-i}(B, B)\right\}-$ $P_{-i}(B, B)=1$.

The above claim establishes that type $\theta$ finds it strictly dominant to use message $D(\theta)$ when he knows that the rival is of type $\theta$.

Claim 2 If $p_{i}(\theta)>0$, we must have $E u_{i}^{\theta}\left(D(\theta) \mid s_{-i}, \bar{\theta}\right)<E u_{i}^{\theta}\left(\overline{D(\theta)} \mid s_{-i}, \bar{\theta}\right)$

Proof Suppose not. Hence, $E u_{i}^{\theta}\left(D(\theta) \mid s_{-i}, \bar{\theta}\right) \geq E u_{i}^{\theta}\left(\overline{D(\theta)} \mid s_{-i}, \bar{\theta}\right)$.

Since $p_{i}(\theta)>0$, we must have

$$
E U_{i}^{\theta}\left(S \mid s_{-i}\right) \geq \max \left[E U_{i}^{\theta}\left(N S, P \mid s_{-i}\right), E U_{i}^{\theta}\left(N S, N \mid s_{-i}\right)\right]
$$

Using Claim 1, we can rewrite this as

$$
\begin{aligned}
& \operatorname{Pr}(\theta) E u_{i}^{\theta}\left(D(\theta) \mid s_{-i}, \theta\right)+\operatorname{Pr}(\bar{\theta}) \max \left[E u_{i}^{\theta}\left(D(\theta) \mid s_{-i}, \bar{\theta}\right), E u_{i}^{\theta}\left(\overline{D(\theta)} \mid s_{-i}, \bar{\theta}\right)\right]-c \\
& \quad>\max \left[E U_{i}\left(N S, D(\theta) \mid s_{-i}\right), E U_{i}^{\theta}\left(N S, \overline{D(\theta)} \mid s_{-i}\right)\right]
\end{aligned}
$$

From our supposition,

$$
\begin{aligned}
L H S & =\operatorname{Pr}(\theta) E u_{i}^{\theta}\left(D(\theta) \mid s_{-i}, \theta\right)+\operatorname{Pr}(\bar{\theta}) E u_{i}^{\theta}\left(D(\theta) \mid s_{-i}, \bar{\theta}\right)-c \\
& =E U_{i}^{\theta}\left(N S, D(\theta) \mid s_{-i}\right)-c
\end{aligned}
$$

Now, $R H S=\max \left\{\begin{array}{c}\operatorname{Pr}(\theta) E u_{i}^{\theta}\left(D(\theta) \mid s_{-i}, \theta\right)+\operatorname{Pr}(\bar{\theta}) E u_{i}^{\theta}\left(D(\theta) \mid s_{-i}, \bar{\theta}\right), \\ \operatorname{Pr}(\theta) E u_{i}^{\theta}\left(\overline{D(\theta)} \mid s_{-i}, \theta\right)+\operatorname{Pr}(\bar{\theta}) E u_{i}^{\theta}\left(\overline{D(\theta)} \mid s_{-i}, \bar{\theta}\right)\end{array}\right\}$

By Claim 1 and our supposition, $R H S=E U_{i}^{\theta}\left(N S, D(\theta) \mid s_{-i}\right)>E U_{i}^{\theta}(N S$, $\left.D(\theta) \mid s_{-i}\right)-c=L H S$, which is a contradiction.

The above claim establishes that whenever there is search with a positive probability, type $\theta$ uses message $\overline{D(\theta)}$ when he knows that the rival is of type $\bar{\theta}$. Claims 1 and 2 determine what actions will be played by a type when the rival type is known. Note that the choice of message post search is independent of the strategy of the rival type. Thus,

$$
E U_{i}^{\theta}\left(S \mid s_{-i}\right)=\operatorname{Pr}(\theta) E u_{i}^{\theta}\left(D(\theta) \mid s_{-i}, \theta\right)+\operatorname{Pr}(\bar{\theta}) E u_{i}^{\theta}\left(\overline{D(\theta)} \mid s_{-i}, \bar{\theta}\right)-c
$$


Claim $3 E U_{i}^{\theta}\left(S \mid s_{-i}\right)-E U_{i}^{\theta}\left(N S, \overline{D(\theta)} \mid s_{-i}\right)=\operatorname{Pr}(\theta)-c$

Proof By Eq. (4), $E U_{i}^{\theta}\left(S \mid s_{-i}\right)-E U_{i}^{\theta}\left(N S, \overline{D(\theta)} \mid s_{-i}\right)$ equals

$$
\begin{aligned}
& \operatorname{Pr}(\theta)\left[E u_{i}^{\theta}\left(D(\theta) \mid s_{-i}, \theta\right)-E u_{i}^{\theta}\left(\overline{D(\theta)} \mid s_{-i}, \theta\right)\right] \\
& \quad+\operatorname{Pr}(\bar{\theta})\left[E u_{i}^{\theta}\left(\overline{D(\theta)} \mid s_{-i}, \bar{\theta}\right)-E u_{i}^{\theta}\left(\overline{D(\theta)} \mid s_{-i}, \bar{\theta}\right)\right]-c
\end{aligned}
$$

By Claim 1, the above expression equals $\operatorname{Pr}(\theta)-c$

Note that $c=\alpha$ or $c=1-\alpha$ are not considered in our range of parameter values. Thus, between search and no search with $\overline{D(\theta)}$, one always strictly dominates the other, based on the values of the parameter. Most importantly, if type $\theta$ searches with positive probability, he will not play the message $\overline{D(\theta)}$ conditional on not searching. If the probability of search is strictly between 0 and 1 , then $D(\theta)$ will be played by $\theta$ conditional on playing action $X=N S$.

Claim $4 E U_{i}^{\theta}\left(S \mid s_{-i}\right)-E U_{i}^{\theta}\left(N S, D(\theta) \mid s_{-i}\right)=\operatorname{Pr}(\bar{\theta})\left(2 P_{-i}(\bar{\theta}, \theta)-1\right)-c$

Proof By Eq. (4), $E U_{i}^{\theta}\left(S \mid s_{-i}\right)-E U_{i}^{\theta}\left(N S, \overline{D(\theta)} \mid s_{-i}\right)$ equals:

$$
\begin{aligned}
& \operatorname{Pr}(\theta)\left[E u_{i}^{\theta}\left(D(\theta) \mid s_{-i}, \theta\right)-E u_{i}^{\theta}\left(D(\theta) \mid s_{-i}, \theta\right)\right] \\
& \quad+\operatorname{Pr}(\bar{\theta})\left[E u_{i}^{\theta}\left(\overline{D(\theta)} \mid s_{-i}, \bar{\theta}\right)-E u_{i}^{\theta}\left(D(\theta) \mid s_{-i}, \bar{\theta}\right)\right]-c
\end{aligned}
$$

By simple algebra, the above expression equals $\operatorname{Pr}(\bar{\theta})\left(2 P_{-i}(\bar{\theta}, \theta)-1\right)-c$.

This claim, along with Claim 3, establishes that when there is search by type $\theta$ of player $i$, we must have $\operatorname{Pr}(\bar{\theta})\left(2 P_{-i}(\bar{\theta}, \theta)-1\right)-c \geq 0$. If there is indifference between search and no search, then the inequality must be satisfied as an equality. Note that this depends on the strategy of the rival candidate.

\subsection{Proof of Lemma 1}

When the rival is known to be bad, the expected payoff of type $G$ from using $P$ is $P_{-i}(B, G)+2\left(1-P_{-i}(B, G)\right)-c$, and that from using message $N$ is $2 P_{-i}(B, G)+$ $\left(1-P_{-i}(B, G)\right)-c$. Thus, the net gain from using $P$ instead of $N$ is $\left(1-2 P_{-i}(B, G)\right)$, which is strictly positive if $P(B, G)<\frac{1}{2}$ and negative if $P_{-i}(B, G)>\frac{1}{2}$. This proves the first part of $(a)$. When the rival is unknown, the payoff to type $G$ from message $P$ is $\alpha\left\{P_{-i}(G, G)+2\left(1-P_{-i}(G, G)\right)\right\}+(1-\alpha)\left\{P_{-i}(B, G)+2\left(1-P_{-i}(B, G)\right)\right\}$ and that from playing $N$ is $\alpha\left\{\left(1-P_{-i}(G, G)\right)\right\}+(1-\alpha)\left\{2 P_{-i}(B, G)+\left(1-P_{-i}(B, G)\right)\right\}$, and the gain from playing $P$ instead of $N$ is $\left[1-2(1-\alpha) P_{-i}(B, G)\right]$, and the second part of $(a)$ follows. The proof of $(b)$ is similar.

With the four basic claims and Lemma 1, we can find the equilibrium, i.e., prove proposition 1 . 


\subsection{Proof of proposition 1}

Part (i): $\alpha<c$

By Claim $4, \alpha<c \Rightarrow E U_{i}^{B}\left(S \mid s_{-i}\right)<E U_{i}^{B}\left(N S, N \mid s_{-i}\right)$

By Claim 3, $1-\alpha>c \Rightarrow E U_{i}^{B}\left(S \mid s_{-i}\right)>E U_{i}^{B}\left(N S, P \mid s_{-i}\right)$

Thus, for type $B$ of either player, using the negative message and not searching strictly dominates other strategies.

Hence, $p_{i}(B)=q_{i}(B)=0 \Rightarrow P_{i}(B, G)=0$ for $i=1,2$. By Lemma 1 part (a), $q_{i}(G)=r_{i}(G)=1$

Also, for each $i, P_{-i}(B, G)=0$ implies

$$
E U_{i}^{G}\left(S \mid s_{-i}\right)-E U_{i}^{G}\left(N S, P \mid s_{-i}\right)=-(1-\alpha)-c<0 \Rightarrow p_{i}(G)=0 .
$$

Part (iii): $\alpha>1-c$

By Claim 4, $1-\alpha<c \Rightarrow E U_{i}^{G}\left(S \mid s_{-i}\right)<E U_{i}^{G}\left(N S, P \mid s_{-i}\right)$

By Claim 3, $\alpha>c \Rightarrow E U_{i}^{G}\left(S \mid s_{-i}\right)>E U_{i}^{G}\left(N S, N \mid s_{-i}\right)$

Thus, for type $G$ of either player, using the positive message while not searching strictly dominates other strategies.

Hence, $p_{i}(G)=0$ and $q_{i}(G)=1 \Rightarrow P_{i}(G, B)=1$. By Lemma 1 part (b), $q_{i}(B)=r_{i}(B)=1$.

By Claim 2, $r_{i}(B)=1 \Rightarrow p_{i}(B)=0$

Part (ii): $c<\alpha<1-c$

Here, $\min (\alpha, 1-\alpha)>c$. Therefore, by Claim 3, for $\theta \in\{G, B\}, E U_{i}^{\theta}\left(S \mid s_{-i}\right)-$ $E U_{i}^{\theta}\left(N S, \overline{D(\theta)} \mid s_{-i}\right)>0$.

Thus, whenever the action $N S$ is played by type $\theta$ with positive probability, $D(\theta)$ is the message employed, or

$$
p_{i}(G)<1 \Rightarrow q_{i}(G)=1 \text { and } p_{i}(B)<1 \Rightarrow q_{i}(B)=0
$$

Next, we claim that we cannot have search with certainty for either type.

Claim 5 For $\alpha \in(1-c, c)$, we cannot have $p_{i}(\theta)=1$ in equilibrium for any $\theta$ for $i=1$ or $i=2$.

Proof Suppose $c<\alpha<1-c$ and $p_{i}(G)=1$. Therefore, $P_{i}(G, B)=r_{i}(G)$, and by Claim 2, $r_{i}(G)=0$. This implies by 1 that $r_{-i}(B)=q_{-i}(B)=0 \Rightarrow P_{-i}(B, G)=0$ (by Lemma 1). Using this in Claim 4 , we have a contradiction, since

$$
E U_{i}^{G}\left(S \mid s_{-i}\right)-E U_{i}^{G}\left(N S, P \mid s_{-i}\right)=-(1-\alpha)-c<0 \Rightarrow p_{i}(G)=0 .
$$

This establishes that we cannot have $p_{i}(G)=1$ in equilibrium for any $i \in\{1,2\}$ if $\alpha \in(1-c, c)$.

Next, suppose that $p_{i}(B)=1$. By Claim 2, we have $r_{i}(B)=1$, implying $P_{i}(B, G)=1$. By Claim 4, this implies that

$$
E U_{-i}^{G}\left(S \mid s_{i}\right)-E U_{-i}^{G}\left(N S, P \mid s_{i}\right)=(1-\alpha)-c>0
$$


In addition to Claim 3 that $E U_{-i}^{G}\left(S \mid s_{-i}\right)-E U_{-i}^{G}\left(N S, P \mid s_{-i}\right)>0$, this implies that $p_{-i}(G)=1$. But we have just seen that this cannot be true in equilibrium.

Equation (5) and Claim 5 together establish that in equilibrium with $c<\alpha<1-c$, we must have $q(G)=1$ and $q(B)=0$. Using this in Claim 4 and Lemma 1, we can show similarly that neither type will have $p(\theta)=0$. Therefore, we must have $p_{i}(\theta) \in(0,1)$ for both types $\theta \in\{G, B\}$ and for both players $i=1,2$. By claim 2, $r_{i}(B)=1$ and $r_{i}(G)=0$.

Also, from Claim 4, we must have for indifference between search and no search for type $\theta$,

$$
\operatorname{Pr}(\bar{\theta})\left(2 P_{i}(\bar{\theta}, \theta)-1\right)=c \Rightarrow P_{i}(\bar{\theta}, \theta)=\frac{1}{2}\left(1+\frac{c}{\operatorname{Pr}(\bar{\theta})}\right)
$$

Since $P_{i}(B, G)=p_{i}(B)$ and $P(G, B)=1-p_{i}(G)$, we are done.

\section{References}

Ansolabehere S, Iyengar S (1995) Going negative: how political advertisements shrink and polarize the electorate. Free Press, New York

Ansolabehere S, Iyengar S, Simon A, Valentino N (1994) Does attack advertising demotivate the electorate? Am Polit Sci Rev 88:829-838

Ansolabehere S, Iyengar S, Simon A (1999) Replicating experiments using aggregate and survey data: the case of negative advertising and turnout. Am Polit Sci Rev 93:901-909

Banks J (1990) A model of electoral competition with incomplete information. J Econ Theory 50:309-325

Berelson Bernard R, Paul L, William MA (1954) Voting: a study of opinion formation in a presidential campaign. University of Chicago Press, Chicago

Brader T (2005) Striking a responsive chord: how political ads motivate and persuade voters by appealing to emotions. Am J Polit Sci 49:388-405

Brooks DJ (2006) The resilient voter: moving toward closure in the debate over negative campaigning and turnout. J Polit 68(3):684-696

Callander S, Wilkie S (2007) Lies, damned lies and political campaigns. Game Econ Behav 60(2):262-286

Desposato S (2008) Going negative in comparative perspective: electoral rules and campaign strategies. In: Working paper, UCSD

Egorov G (2012) Single-issue campaigns and multidimensional politics. In: Working paper, Northwestern University

Finkel S, Geer J (1998) A spot check: casting doubt on the demobilizing effect of attack advertising. Am J Polit Sci 42:573-595

Freedman P, Goldstein KM (1999) Measuring media exposure and the effects of negative campaign ads. Am J Polit Sci 3:1189-1208

Freedman P, Goldstein KM (2002) Campaign advertising and voter turnout: new evidence for a stimulation effect. J Polit 64:721-740

Geer J (2006) In defense of negativity: attack ads in presidential campaigns. University of Chicago Press, Chicago

Harrington JJ, Hess GD (1996) A spatial theory of positive and negative campaigning. Game Econ Behav 17:209-229

Kahn KF, Kenney P (1999) Do negative campaigns mobilize or suppress turnout? Clarifying the relationship between negativity and participation. Am Polit Sci Rev 93:877-890

Kaplan N, Park D, Ridout T (2006) Dialogue in US senate campaigns? An examination of issue discussion in candidate television advertising. Am J Polit Sci 50(3):724-736

Kelley S Jr. (1960) Political campaigning: problems in creating an informed electorate. The Brookings Institution, Washington 
Lau R, Sigelman L, Heldman C, Babbitt P (1999) The effects of negative political advertisements: a metaanalytic assessment. Am Polit Sci Rev 93(4):851-875

Lau R, Sigelman L, Rovner IB (2007) The effects of negative political campaigns: a meta-analytic reassessment. J Polit 69:1176-1209

Mattes K (2007) Attack politics: who goes negative and why? In: Caltech social sciences working paper 1256

Petrocik JR (1996) Issue ownership in presidential elections, with a 1980 case study. Am J Polit Sci 40(3):825-850

Prat A (2004) Rational voters and political advertising. In: Weingast B, Wittman D (eds) Oxford handbook of political economy, Oxford University Press, Oxford

Polborn M, Yi DT (2006) Informative positive and negative campaigning. Q J Polit Sci 1(4):351-371

Sellers PJ (1998) Strategy and background in congressional campaigns. Am Polit Sci Rev 92(1):159-171

Simon AF (2002) The winning message: candidate behavior, campaign discourse and democracy. Cambridge University Press, Cambridge

Skaperdas S, Grofman B (1995) Modelling negative campaigning. Am Polit Sci Rev 89:49-61

Snyder J (1989) Election goals and the allocation of campaign resources. Econometrica 57(3):637-660

Wattenberg M, Brians C (1999) Negative campaign advertising: demobilizer or mobilizer? Am Polit Sci Rev 93:891-900 